\title{
Increased Detection Rate of Prevotella Intermedia among Pregnant Periodontitis Patients in Romania
}

\author{
Igor Jelihovschi ${ }^{1}$, Olivia Simona Dorneanu*1, Ralucaloana Serban ${ }^{1}$, Aida Corina Badescu ${ }^{1}$, Ramona \\ Gabriela Ursu' ${ }^{1}$, Ioana Alexandra Martu², Sorina Mihaela Solomon², Luminita Smaranda Iancu ${ }^{1}$
}

${ }^{1}$ Department of Preventive Medicine and Interdisciplinary, Grigore T. Popa University of Medicine and Pharmacy, Romania

${ }^{2}$ Department of Odontology-Periodontology, Grigore T. Popa University of Medicine and Pharmacy, Romania

Received: January 03, 2018; Published: January 10, 2018

*Corresponding author: Olivia Simona Dorneanu, AssociateProfessor, Phd, Microbiology, Department of Preventive Medicine and Interdisciplinarity,Grigore T. Popa University of Medicine and Pharmacy, Romania, Email: olivia.dorneanu@umfiasi.ro

Abstract

Associated to pregnancy hormonal variations in plasma are favorable conditions to developing or exacerbating preexistent periodontitis. Pregnancy associated periodontal inflammation is correlated with or correlates with increasing abundance of Prevotella intermedia in the periodontal pocket. In the present study we quantified by qPCR the subgingival counts of total bacteria, P. intermedia and Porphyromonas gingivalis in pregnant women, non-pregnant and male comparatively to assess the distribution patterns of $P$. intermedia in periodontitis patients according to the pregnancy status of the host. P. intermedia is detected more frequently and in greater abundance in pregnant periodontitis patients.

Keywords: Periodontal disease; qPCR; Pregnancy; Prevotella intermedia

\section{Introduction}

The oral cavity is heavily colonized by more than 700 different microbial species forming a dynamic and complex ecosystem. The members of the ecosystem establish symbiotic relationships with the host which is subjected to continuous variation depending on the diet, lifestyle and general health status of the host [1-3]. There is a thin balance between peaceful coexistence of oral microbial populations on oral epithelia promoting tissue protection from xenobiotics and harmful to the host interactions with the members of oral microbiota. In case of such dysbiotic relationships members of the oral microbiota can lead to activation of immune cells provoking inflammatory chronic oral diseases. Periodontitis is an excellent example of such disease [4,5]. Periodontal disease represents a chronic, inflammatory condition of tooth supporting tissues and the final outcome is the spontaneous avulsion oh the teeth from the dental alveoli if left untreated. A high number of factors are involved in the etiology of periodontitis which are host dependent, oral environment dependent and microbial community dependent [1,6]. Several host dependent factors can influence the transition from healthy mutualist or commensal microbial communities to disease provoking communities which benefits from the subgingival inflammatory microenvironment. Despite the fact that oral microbiome is the most studied microbiome in terms of composition and function, our understanding of periodontitis has not changed significantly in the last years and development of predictable biomarkers for early diagnosis or therapy outcome are still insufficient $[7,8]$.
Several bacterial species, members of Socransky's red complex are strongly associated with the development of periodontal disease therefore their detection and quantification are used in diagnosis and therapy planning purposes. Prevotella intermedia and Porphyromonas gingivalis are Gram negative, anaerobes and act as pathobionts which although in low abundance in the gingival sulcus but through special synergistic actions can influence negatively the normal microbiome composition and facilitate the development of an dysbiotic inflammation provoking microbiota. Such inflammatory phenomena are amplified by the rise of blood hormones during pregnancy $[9,10]$. Steroid sex hormones target the gingival and deep periodontal tissues leading to increased vasculature, elevated proinflammatory cytokines, decreased epithelium keratinization and gingival tissues permeability resulting in exaggerated gingival edematous response to subgingival bacteria. In addition, hormonal variation act as a selective factor for bacterial growth in the subgingival environment and elevated subgingival counts of $P$. intermedia are found due to the interactions between female sex hormones with the fumarate reductase system $[11,12]$.

During pregnancy associated periodontal inflammation, oral bacteria, especially those which higher invasive capabilities such as Prevotella intermedia and Porphyromonas gingivalis, can translocate into the bloodstream and cause inflammation at distant sites. Periodontal disease has been associated with negative birth outcomes. In the study conducted by Swati et al. [13] on women with preeclampsia, a higher proportion of $P$. intermedia 
and $P$. gingivalis positive cases comparative to control cases was found. In the same study, the authors clearly demonstrated a causal relationship between occurrence of preeclampsia during pregnancy with periodontal disease by simultaneous detection of periodontal pathogens in periodontal pockets and placenta by the means of qPCR [13].

Collection of qualitative subgingival samples from pregnant women imposes several difficulties due to the exaggerated edematous gingival response and abundant gingival bleeding to any mechanical stimulus. Sampling of subgingival bacteria by insertion of paper points in the gingival sulcus may impose negative influences on the detection and quantification of periodontal pathogens. Therefore sampling by the means of sterile Gracey curetes seems to be a much better sampling alternative. In the current study we measured the subgingival counts of $P$. intermedia and $P$. gingivalis by the means of qPCR in pregnant women with periodontitis comparative to non-pregnant women and men $[14,15]$.

\section{Aim of the Study}

The purpose of the present study is to investigate the associations of subgingival counts of $P$. intermedia and $P$. gingivalis with the periodontal status of pregnant women which may uncover particularities in the composition of subgingival microbial community among this special category of periodontitis patients directly influencing the treatment planning.

\section{Materials and Methods}

For the purpose of the study 42 pregnant women with age range between 21-37 years suffering from periodontitis were recruited from the university periodontology clinic of Grigore T. Popa University of Medicine and Pharmacy and served as the test population group. As control cases we used two population groups one group consisting of 35 periodontitis women who had never been pregnant and didn't use oral contraceptives as method of contraception. The second control group consisted of 28 men diagnosed with chronic periodontitis. This study was approved by the ethic commission of Grigore T. Popa University of Medicine and Pharmacy, Iasi and each patient gave the written consent for enrolment in the present study, denial of signing the consent led to automatic exclusion from the study. Patients with other systemic diseases which are known risk factors for developing periodontal disease (except of the physiologic status of pregnancy) such as cardio-vascular diseases, diabetes, any form of immunodeficiency, periodontal treatment in the last 2 years or antibiotic administration 6 months prior to clinical examination and specimen collection were not included in the present study.

All subjects were diagnosed according to criteria described by the American Academy of Periodontology [16]. The study participants were examined and diagnosed by two specialized and accredited periodontologists which underwent calibration to exclude inter- and intra-examiner variation. In a group of 7 patients who did not participate in the present study pairs of examinations were conducted on each patient and the results were analyzed by the means of k-test. The intra- and inter-examiner coefficients ranged between 0.78 and 0.85 for PD and CAL. All the patients were subjected to standard periodontal clinical examination and the following indices were recorded: (i) BOP, (ii)PD and (iii)CAL. Subgingival samples collection.

Samples were collected from the four deepest periodontal pockets from each maxillary and mandibular semi-arch and pooled into one $2 \mathrm{~mL}$ Eppendorf tube and stored at $-80^{\circ} \mathrm{C}$ until analyses. Before sampling each tooth was air dried and isolated from saliva by cotton rolls. Because collection of subgingival bacteria by absorbable paper points in pregnant patients can introduce biases in microbiological investigations due to excessive bleeding, sampling by appropriate Gracey periodontal curettes was preferred.

The periodontal curette was inserted into the periodontal pocket with the active part as deep as possible and the plaque was collected as a single stroke on the dental root surface. The working end of the curette was wiped off with one paper point into a $2 \mathrm{~mL}$ Eppendorf tube.

\section{DNA Extraction}

DNA extractions were performed with the QIAamp DNA Mini Kit (Qiagen, Germany) following manufacturer instructions for Gram positive bacteria in order to be able to quantify the total bacterial load in subgingival plaque samples. After extraction, the yield and the purity of DNA was assessed by NanoPhotometer ${ }^{\circledR}$ (Implen Gmbh, Germany) readings of optical densities (OD) at $260 \mathrm{~nm}$ and $280 \mathrm{~nm}$. The ratio of $\mathrm{OD}_{260} / \mathrm{OD}_{280}$ was used as an indicator of the DNA purification efficiency, and values of 1.7-2.0 represents acceptable DNA purity for PCR amplification.

\section{Quantitative PCR Assays}

In order to quantify the total bacterial load a real-time PCR assay using a double stranded DNA dye. The reaction composition was: $10 \mu \mathrm{L}$ of GoTaq ${ }^{\circledR}$ qPCR Master Mix solution (Promega, USA), $30 \mathrm{nM}$ final concentration ROX reference dye, $200 \mathrm{nM}$ primers final concentration, $1 \mu \mathrm{L}$ of target DNA and up to $20 \mu \mathrm{L}$ PCR-grade water. The following primers were used, 5'-TCCTACGGGAGGCAGCAGT-3' and 5'-GGACTACCAGGGTATCTAATCCTGTT-3'. The quantification was performed according to a constructed standard curve plasmid-based, amplicon sequence inserted (Primerdesign, UK). Thermal cycling conditions were as follow: initial denaturation at $95^{\circ} \mathrm{C}-10$ min, 40 cycles at $95^{\circ} \mathrm{C}-10 \mathrm{~s}, 65^{\circ} \mathrm{C}-15 \mathrm{~s}$ and $72^{\circ} \mathrm{C}-40 \mathrm{~s}$. For quantification of $P$. gingivalis and $P$. intermedia and, a Taq Man probe assay was employed using a GoTaq ${ }^{\circledR}$ Probe qPCR Master Mix solution. We employed the same sequence of primers and probes, as well as the same reaction conditions as indicated by Hyvärinen et al. [17].

\section{Results}

The proportion of positive cases and the average bacterial counts according to study groups can be founded in (Table 1) and (Figures $1 \& 2$ ). The average counts for total bacteria varies according to study group and the test group presents an average bacterial count of $2.17 \times 10^{11}$ comparative to lower bacterial counts of $3.71 \times 10^{10}$ and $4.27 \times 10^{10}$ for the non-pregnant women and men accordingly. The 
difference of the average bacterial counts among the study groups is statically significant, $\mathrm{p}$ value of ANOVA test is 0.001 . The counts of $P$. gingivalis and P. intermedia on average were found to vary among the different study groups although the differences did not show any statistical significance (Table 1). There is an obvious difference in mean counts of $P$. intermedia between the test group and control groups, the test group presenting higher mean value of bacterial count. The difference has not any statistical significance but is very obvious in order to be avoided. The proportion of positive cases patients with P. intermedia was $83.4 \%, 71.4 \%$ and $46.4 \%$ in the test group and control groups respectively. The difference in the positive cases with $P$. intermedia among the study groups is statistically significant, $\mathrm{p}=0.005$. The differences between the study groups in positive cases with $P$. gingivalis show no statistical significance.

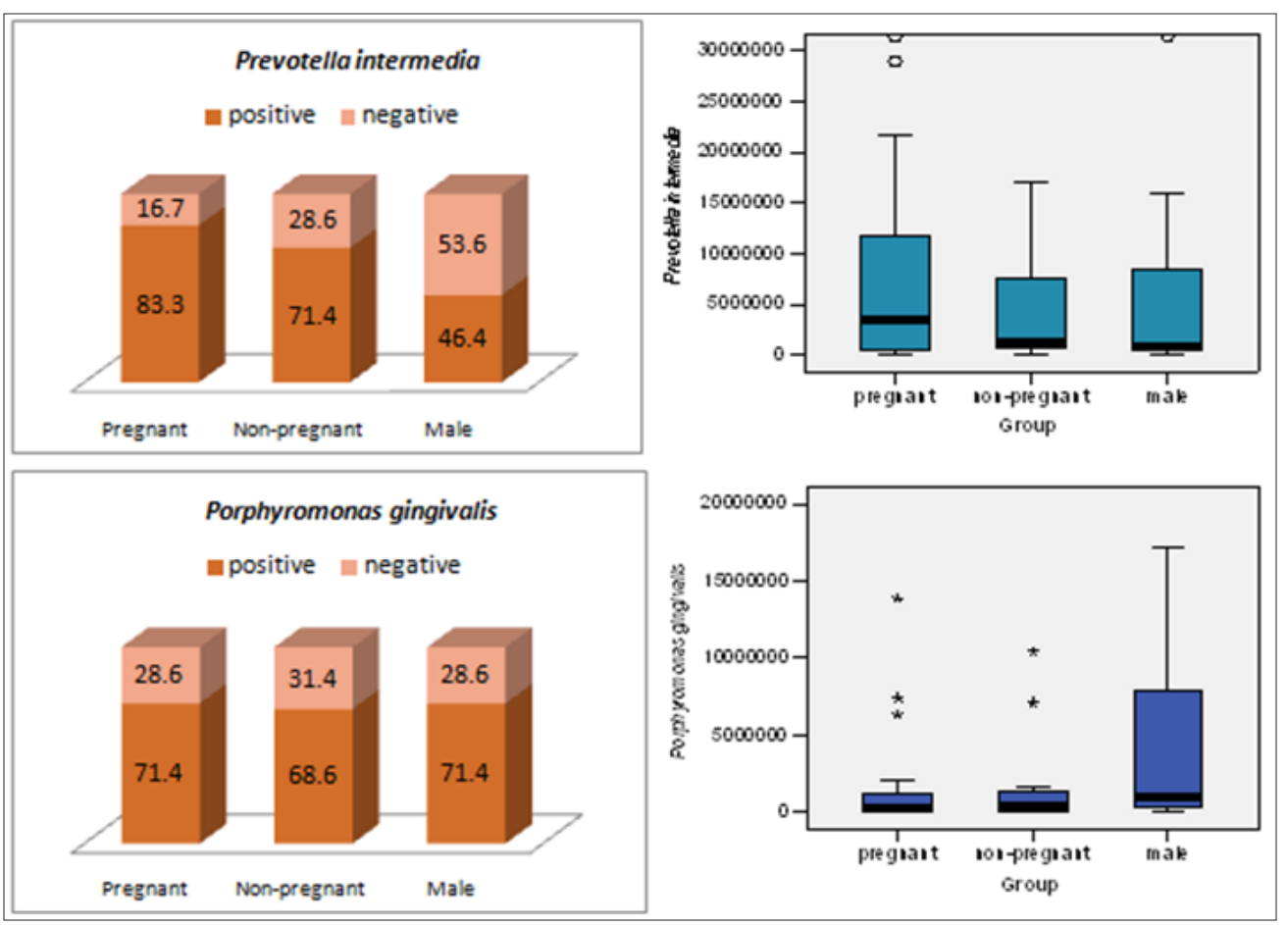

Figure 1: Detection rate and average counts of total bacteria, Porphyromonas gingivalis and Prevotella intermedia.

Table 1: Periodontal pathogens counts according to the study group. The values are expressed as mean values and range.

\begin{tabular}{|c|c|c|c|c|}
\hline Group & P. intermedia & P. gingivalis & Positive & Total Bacteria \\
\hline $\begin{array}{l}\text { Pregnancy } \\
(\mathrm{n}=45)\end{array}$ & $\begin{array}{c}1.67 \times 10^{7} \\
\left(9.28 \times 10^{2}-3.23 \times 10^{8}\right)\end{array}$ & $\begin{array}{c}3.53 \times 106 \\
\left(2.16 \times 10^{2}-6.52 \times 10^{7}\right)\end{array}$ & $\begin{array}{l}\mathrm{P} . \mathrm{g}=30 \\
\mathrm{P} . \mathrm{i}=35\end{array}$ & $\begin{array}{c}2.17 \times 1011 \\
\left(6.71 \times 10^{9}-9.53 \times 10^{11}\right)\end{array}$ \\
\hline $\begin{array}{l}\text { Non-pregnancy } \\
\qquad(\mathrm{n}=35)\end{array}$ & $\begin{array}{c}7.51 \times 10^{6} \\
\left(1.74 \times 10^{4}-8.87 \times 10^{7}\right)\end{array}$ & $\begin{array}{c}5.63 \times 10^{6} \\
\left(1.92 \times 10^{2}-6.4 \times 10^{7}\right)\end{array}$ & $\begin{array}{l}\mathrm{P} . \mathrm{g}=24 \\
\mathrm{P} . \mathrm{i}=25\end{array}$ & $\begin{array}{c}3.71 \times 1010 \\
\left(1.1 \times 10^{7}-2.53 \times 10^{11}\right)\end{array}$ \\
\hline $\begin{array}{c}\text { Men } \\
(n=28)\end{array}$ & $\begin{array}{c}6.81 \times 10^{6} \\
\left(1.45 \times 10^{7}-3.14 \times 10^{5}\right)\end{array}$ & $\begin{array}{c}8.48 \times 106 \\
\left(1.45 \times 10^{3}-6.4 \times 10^{7}\right)\end{array}$ & $\begin{array}{l}P . g=20 \\
P . i=13\end{array}$ & $\begin{array}{c}4.27 \times 10^{10} \\
\left(4.16 \times 10^{7}-4.83 \times 10^{11}\right)\end{array}$ \\
\hline $\mathrm{p}$ valuefor $\mathrm{F}_{\text {Anova }}$ test & 0.595 & 0.042 & & 0.001 \\
\hline
\end{tabular}

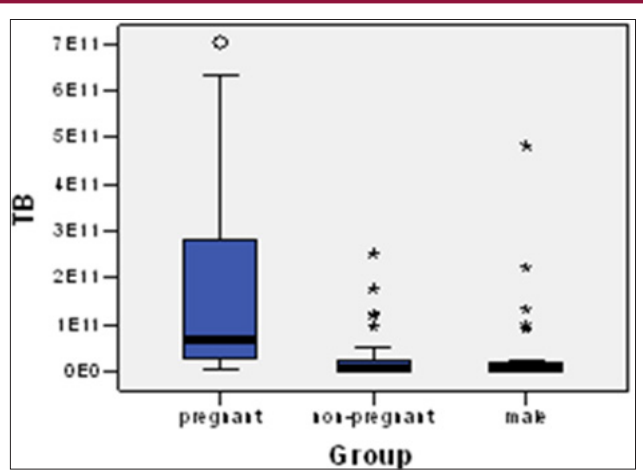

Figure 2: Total subgingivalbacteria counts according to host status.

\section{Discussion}

The highly specialized studies involving next generation sequencing under covers the huge impact on the health status of the human microbiome. The Human Microbiome Project revealed the truly diversity of the oral microbiome between subjects and the bacterial composition rather than specific species account for oral infections [18]. Periodontal disease is initiated by dysbiotic changes of the oral microbiome and the tiny microbial cells control not only the oral health but also other systemic diseases. Associations of periodontal disease with heterogeneous diseases as atherosclerosis, myocardial infarction, stroke, diabetes and adverse pregnancy outcomes. Periodontitis in pregnancy has been correlated with preterm birth, low birth weight, miscarriage and 
preeclamsia [19-21]. The host environment can modulate the diversity of the microbial cells colonizing the oral mucosal surfaces. The physiologic status of pregnancy is accompanied by variation in plasma hormonal levels inducing alterations in cell composition of oral microbiota especially decreasing the abundance of species belonging to Neisseria and Aggregatibacter genus [22].

Several authors studying subgingival bacterial profile of pregnancy induced gingivitis and periodontitis have shown increased proportion of fusobacteria, Campylobacter spp., Tannerella forsythia, Prevotella nigrescens, Prevotella intermedia and other periodontal pathogens [23-26]. In the present study to emphasize the difference in pregnancy associated periodontitis as control groups we used also an age-matched population group composed of male periodontitis patients. In terms of both positive cases and mean counts there is an evident correlation of $P$. intermedia with pregnancy induced periodontitis. The proportion of positive cases in pregnancy group is higher comparative to non-pregnant women $(83.3 \%$ vs $71.4 \%)$ and the difference is much more obvious comparative to the male patients group in which $46.6 \%$ of patients tested positive for $P$. intermedia. When comparing the proportion of positive cases for $P$. gingivalis the differences are much diminished. The central finding of the present study was the much higher mean counts of total bacteria and P. intermedia in the pregnant women suffering from periodontitis while there is no evident difference in the mean of $P$. gingivalis according to the study group. A next generation sequencing study on pregnant women without periodontitis failed to detect notorious periodontal pathogens emphasizing the role of these bacterial species in developing periodontitis and can be used as biomarkers to diagnose pregnancy associated periodontitis [22].

\section{Conclusion}

In the present study we managed to highlight the differences of preferential colonization of pregnant women suffering from periodontitis in higher proportions and abundance by P. intermedia. Studying the microbiome of pregnancy induced periodontitis more biological insights relevant to treatment and prevention of periodontitis may be uncovered.

\section{References}

1. Baker JL, Bor B, Agnello M, Shi W, He X (2017) Ecology of the Oral Microbiome: Beyond Bacteria. Trends Microbiol 25(5): 362-374.

2. Dewhirst FE, Chen T, Izard J, Paster BJ, Tanner ACR, et al. (2010) The Human Oral Microbiome. J Bacteriol 192(19): 5002-5017.

3. Grassl N, Kulak NA, Pichler G, Geyer PE, Jung J, et al. (2016) Ultradeep and quantitative saliva proteome reveals dynamics of theoral microbiome. Genome Med 8(1): 44.

4. Costalonga M, Herzberg MC (2014) The oral microbiome and the immunobiology of periodontal disease and caries. Immunol Lett 162(2): 22-38.

5. Yamanaka W, Takeshita T, Shibata Y, Matsuo K, Eshima N, et al. (2012) Compositional Stability of a Salivary Bacterial Population against Supragingival Microbiota Shift following Periodontal Therapy. PLoS One $7(8): 42806$.

6. Drisko CL (2014) Periodontal debridement: still the treatment of choice. J Evid Based Dent Pract 14: 33-41.
7. Preshaw PM, Holliday R, Law H, Heasman PA (2013) Outcomes of nonsurgical periodontal treatment by dental hygienists in training: impact of site- and patient-level factors. Int J Dent Hyg 11(4): 273-279.

8. Lamont RJ, Hajishengallis G (2015) Polymicrobial synergy and dysbiosis in inflammatory disease. Trends Mol Med 21(3): 172-183.

9. Hajishengallis G, Darveau RP, Curtis MA (2012) The keystone-pathogen hypothesis. Nat Rev Microbiol 10(10): 717-725.

10. Haffajee AD, Socransky SS (1994) Microbial etiological agents of destructive periodontal diseases. Periodontol 2000 5: 78-111.

11. Yokoyama M, Hinode D, Yoshioka M, Fukui M, Tanabe S, et al. (2008) Relationship between Campylobacter rectus and periodontal status during pregnancy. Oral MicrobiolImmunol 23(1): 55-59.

12. Adriaens LM, Alessandri R, Spörri S, Lang NP, Persson GR (2009) Doe's pregnancy have an impact on the subgingival microbiota? J Periodontol 80(1): 72-81.

13. Swati P, Thomas B, Vahab SA, Kapaettu S, Kushtagi P (2012) Simultaneous detection of periodontal pathogens in subgingival plaque and placenta of women withhypertension in pregnancy. Arch Gynecol Obstet 285(3): 613-619.

14. Nguyen-Hieu T (2013) Microbial sampling process can change results of microbiological analysis in periodontitis diagnosis. A minireview J Investig Clin Dent 4(3): 151-159.

15. Costalonga M, Herzberg MC (2014) The oral microbiome and the immunobiology of periodontal disease and caries. Immunol Lett 162(2): 22-38.

16. Armitage GC (1999) Development of a classification system for periodontal diseases and conditions. Ann Periodontol 4(1): 1-6.

17. Hyvärinen K, Laitinen S, Paju S, Hakala A, Suominen-Taipale L, et al. (2009) Detection and quantification of five major periodontalpathogens by single copy gene-based real-time PCR. Innate Immun 15(4): 195-204.

18. (2012) Structure, function and diversity of the healthy human microbiome. Human Microbiome Project Consortium, Nature 486: 207214.

19. Tonetti MS, Van Dyke TE (2013) working group 1 of the joint EFP/AAP workshop.Periodontitis and atherosclerotic cardiovascular disease: consensus report of theJoint EFP/AAP Workshop on Periodontitis and Systemic Diseases. J Periodontol 84(4): 24-29.

20. Cardoso EM, Reis C, Manzanares-Céspedes MC (2017) Chronic periodontitis,inflammatory cytokines, and interrelationship with other chronic diseases. Postgrad Med 130(1): 98-104.

21. Luchian I, Martu I, Ioanid N, Goriuc A, Vata I, et al. (2016) Salivary IL-1ß: A Biochemical Marker that Predicts Periodontal Disease in Orthodontic Treatment. Rev Chim (Bucharest) 67(12): 2479-2483.

22. Paropkari AD, Leblebicioglu B, Christian LM, Kumar PS (2016) Smoking, pregnancy and the subgingival microbiome. Sci Rep 6: 30388.

23. Muramatsu Y, Takaesu Y (1994) Oral health status related to subgingival bacterial flora and sex hormones in saliva during pregnancy. Bull Tokyo Dent Coll 35(3): 139-151.

24. Jensen J, Liljemark W, Bloomquist C (1981) The effect of female sex hormones on subgingival plaque. J Periodontol 52(10): 599-602.

25. Adriaens LM, Alessandri R, Spörri S, Lang NP, Persson GR (2009) Does pregnancy have an impact on the subgingival microbiota? J Periodontol 80(1): 72-81.

26. Gursoy M, Haraldsson G, Hyvönen M, Sorsa T, Pajukanta R, et al. (2009) Does the frequency of Prevotella intermedia increase during pregnancy? Oral Microbiol Immunol 24(4): 299-303. 


$\begin{array}{ll}\text { BIOMEDICAL } & \text { Assets of Publishing with us } \\ \text { RESEARCHES } & \text { - Global archiving of articles }\end{array}$

